\title{
DEGRADAÇÃO FOTOELETROQUÍMICA DE CORANTES DISPERSOS EM EFLUENTE TÊXTIL UTILIZANDO FOTOANODOS DE Ti/TiO
}

Tamara F. T. Brunelli, Thaís T. Guaraldo, Fabiana M. M. Paschoal e Maria Valnice B. Zanoni*

Departamento de Química Analítica, Instituto de Química, Universidade Estadual Paulista, CP 355, 14801-970 Araraquara - SP, Brasil

Recebido em 6/12/07; aceito em 6/8/08; publicado na web em 15/12/08

\begin{abstract}
PHOTOELETROCHEMICAL DEGRADATION OF DISPERSE DYES IN TEXTILE EFFLUENT ON PHOTOANODES OF $\mathrm{Ti} / \mathrm{TiO}_{2}$. The degradation of disperses dyes in aqueous solution and in effluents from textile industry has been investigated by photoelectrocatalytic oxidation using nanoporous thin films electrodes of $\mathrm{Ti} / \mathrm{TiO}_{2}$. Samples of dispersil black dye and dispersil blue dye after 300 min of photoelectrolyzed at applied potential of $+1.0 \mathrm{~V}$ and UV irradiation exhibited $100 \%$ of discoloration and $90 \%$ and $64 \%$ reduction total organic carbon, respectively. The proposed method was applied with success in a textile industry effluent containing residues of these dyes, which after $300 \mathrm{~min}$ of treatment leads to reduction of $60 \%$ of COD and $64 \%$ removal of TOC.
\end{abstract}

Keywords: disperse dye; textile effluent; photoeletrochemistry.

\section{INTRODUÇÃO}

A contaminação de águas têm sido um dos grandes problemas da sociedade moderna e de acordo com previsões da Companhia de Saneamento Básico do Estado de São Paulo (SABESP), estima-se que a partir de 2010 a demanda de água será superior à capacidade hidráulica dos mananciais do estado. ${ }^{1}$ Deste modo, a água de descarte, oriunda dos processos da indústria têxtil, tem sido motivo de grande preocupação nas últimas décadas, devido ao grande volume de água requerido e à grande quantidade de corantes orgânicos sintéticos e outros aditivos químicos presentes em seus resíduos. ${ }^{2}$ Esses efluentes se não forem tratados de maneira adequada, antes de serem lançados em águas naturais, podem atingir reservatórios e estações de tratamento de água tornando-se um sério risco ambiental e à saúde. ${ }^{3}$

Dentre as várias classes de corantes sintéticos utilizados na indústria têxtil, os corantes dispersos merecem atenção. São compostos aromáticos não iônicos, na maioria contêm grupos azo como cromóforo, são pouco solúveis em água e altamente utilizados na tintura de fibras sintéticas, tais como poliéster, triacetato de celulose e poliamidas. A adição de agentes dispersantes durante a etapa de tintura sob alta temperatura $\left(80^{\circ} \mathrm{C}\right)$ é uma das responsáveis pelo transporte do corante à fibra hidrofóbica, ${ }^{4,5}$ cuja etapa tem mudado a concepção desses corantes como prováveis poluentes de águas superficiais. ${ }^{6,7}$ Esses corantes formam sistemas de micelas estáveis nestas soluções, que são facilmente transportadas em meio aquoso, contribuindo para sua presença em águas superficiais, para sua acumulação em sedimentos, solos e conseqüente contaminação da água potável oriunda de estações de tratamento de água. ${ }^{6}$ A preocupação com rejeitos contendo essa classe de corantes tem crescido nos anos mais recentes, devido a suas comprovadas propriedades mutagênicas. ${ }^{8-17}$

Os métodos de tratamento para corantes dispersos baseados em processos biológicos, físico-químicos e utilização de ozônio têm sido inadequados para sua remoção. ${ }^{18-20}$ Métodos de descoloração de corantes dispersos baseados em processos fotoquímicos têm alcançado apenas $55 \%$ de eficiência. ${ }^{21}$ Melhores resultados para descoloração são descritos para processos usando eletroquímica,

*e-mail: boldrinv@iq.unesp.br hipoclorito e reagente de Fenton, ${ }^{22}$ no entanto, a mineralização final desses corantes não é descrita.

A técnica de fotoeletrocatálise tem sido aplicada com o objetivo de aumentar a eficiência de processos fotocatalíticos pela combinação das vantagens da fotocatálise com eletrocatálise. ${ }^{8-12}$ A velocidade típica das reações num semicondutor varia de fentossegundos para o aprisionamento de cargas, a milissegundos para transferência interfacial, sendo esta última a etapa determinante da velocidade. $\mathrm{O}$ uso de um fotocatalisador como fotoanodo, onde se pode aplicar um potencial sob iluminação de luz ultravioleta, pode aumentar a eficiência do processo fotocatalítico, retardando a recombinação de cargas nas partículas do semicondutor. Este método tem sido utilizado com sucesso para a degradação de vários compostos orgânicos. ${ }^{13-17}$

Neste trabalho estudou-se a oxidação fotoeletrocatalítica de um efluente coletado de uma indústria têxtil usando eletrodos de $\mathrm{Ti} / \mathrm{TiO}_{2}$ preparados pelo método sol-gel, sob irradiação UV. O método foi ainda testado para os corantes usados na composição do banho de tintura no mesmo dia em que o efluente foi coletado. Os parâmetros cinéticos de descoloração e degradação foram avaliados através das análises espectrofotométricas na região do UV-Visível, remoção do carbono orgânico total (COT) e análise cromatográfica com detector de arranjo de diodos.

\section{PARTE EXPERIMENTAL}

\section{Preparo do eletrodo de filme fino de $\mathrm{Ti} / \mathrm{TiO}_{2}$}

Tetraisopropóxido de titânio (Aldrich) foi usado como precursor para o preparo da suspensão coloidal de $\mathrm{TiO}_{2}$. Alíquota de $20,0 \mathrm{~mL}$ de tetraisopropóxido de titânio foi adicionada a uma solução de ácido nítrico em água deionizada (Milli-Q) de tal modo que a razão molar seguiu a seguinte relação: Ti: $\mathrm{H}^{+}: \mathrm{H}_{2} \mathrm{O}(1: 0,5: 200)$. O precipitado resultante foi agitado continuamente para obtenção de uma suspensão estável e então submetido à diálise em água deionizada até $\mathrm{pH}$ 3,5, utilizando-se uma membrana Micropore (3500 MW). O filme fino de $\mathrm{TiO}_{2}$ foi depositado, imergindo o substrato (chapas de titânio metálico de 0,5 mm de espessura, Goodfellow Cambridge Ltda.) na suspensão do semicondutor e retirando-o a uma velocidade constante, controlada mecanicamente por uma polia. Depois de seco, o eletrodo 
foi calcinado a $350{ }^{\circ} \mathrm{C}$ por $3 \mathrm{~h}$, completando um ciclo. Quatro ciclos adicionais foram completados e o eletrodo estava pronto para ser usado, de acordo com o procedimento descrito anteriormente. ${ }^{13-16}$

\section{Instrumentação e procedimento de análise}

Os experimentos de fotoeletrocatálise foram realizados em um reator com capacidade para $250 \mathrm{~mL}$. Na célula foi posicionado o eletrodo de trabalho, um eletrodo auxiliar e como referência o eletrodo de $\mathrm{Ag} / \mathrm{AgCl}$ imerso em um capilar de Luggin. A área fotoativa do anodo $\left(\mathrm{TiO}_{2}\right)$ foi $12 \mathrm{~cm}^{2}$, que foi iluminado usando-se uma lâmpada de vapor de mercúrio da Philips de $125 \mathrm{~W}\left(\mathrm{I}=9,23 \mathrm{~W} / \mathrm{m}^{2}\right)$ sem o bulbo de vidro, inserida num tubo de quartzo posicionado a uma distância de $5 \mathrm{~cm}$ do fotoanodo. $\mathrm{O}$ sistema foi refrigerado por banho termostatizado (Nova Técnica, Brasil) pela circulação externa de água. As soluções de corante e efluente foram colocadas no reator sem nenhum pré-tratamento e o tratamento fotoeletrocatalítico foi realizado com borbulhamento de ar. O eletrodo de rede de platina foi usado como contra-eletrodo.

Um potenciostato/galvanostato da marca EG\&G Instruments (Princeton Applied Research) modelo 283, controlado pelo software eletroquímico 270 , foi utilizado nas medidas de fotoeletrocatálise.

A amostra do efluente foi coletada de uma planta de efluente específico da Indústria Malhas Vêneto, localizada em Farroupilha, Rio Grande do Sul, em frasco de polietileno e mantida sob refrigeração a $4{ }^{\circ} \mathrm{C}$. A amostra do efluente foi submetida ao tratamento fotoeletrocatalítico sem qualquer pré-tratamento. A característica da composição do banho de tintura resultando no efluente está descrita a seguir: composição do efluente - efluente coletado no mesmo dia do banho de tintura foi otimizado com: $50 \mathrm{ppm}$ do corante dispersil preto e $50 \mathrm{ppm}$ do corante dispersil azul, 1500 ppm de bórax e 800 ppm de agente dispersante denominado "emulsogen".

As concentrações dos corantes preto dispersil e azul dispersil em amostras em meio aquoso e do efluente têxtil em solução foram monitoradas pelas medidas de absorbância das amostras em intervalo de tempo controlado, usando-se um espectrofotômetro Hewlett Packard 8453 operando entre 190-800 nm em célula de quartzo. A análise de carbono orgânico total (COT) foi monitorada usando-se o analisador de carbono orgânico (Shimadzu 5000A).

As medidas de $\mathrm{pH}$ do efluente foram realizadas em um $\mathrm{pHmetro}$ Corning 555, equipado com um eletrodo combinado de vidro.

As medidas de condutividades foram realizadas em um condutivímetro Crison, modelo GLP 32. A determinação da demanda química de oxigênio (DQO) foi feita através do método do refluxo com dicromato (Standard Methods 5220B). ${ }^{23}$

Um cromatógrafo líquido de alta eficiência Shimadzu Model 10 AVP acoplado a um detector de arranjo de diodos foi usado para separar e identificar produtos e intermediários das soluções fotoeletrocatalisadas dos corantes e do efluente. Uma coluna de separação C-18 (4.6 mm x $250 \mathrm{~mm}, 5 \mu \mathrm{m})$ e fase móvel de acetonitrila/água $(80: 20(\mathrm{v} / \mathrm{v}))$ com fluxo de $1,0 \mathrm{~mL} \mathrm{~min}^{-1}$ foram utilizadas. Os sinais obtidos do detector foram analisados pela integração da área. Os procedimentos foram feitos em triplicada para cada amostra.

\section{RESULTADOS E DISCUSSÃO}

A fim de testar a efetividade do processo de oxidação fotoeletrocatalítica do efluente, foi investigado, previamente, sua performance na remoção de cada corante componente da mistura usada no banho de tintura, comparando-se a remoção da cor, detecção cromatográfica e remoção do COT para os corantes comerciais: preto dispersil e azul dispersil, os quais são corantes azo com estruturas químicas protegidas por patente.

\section{Oxidação fotoeletrocatalítica dos corantes preto e azul dispersil}

A Figura 1 apresenta espectros de absorção típicos para 50 ppm do corante preto dispersil (DPD) (Curva A) e $50 \mathrm{ppm}$ do corante azul dispersil (DAD) (Curva B) em $0,1 \mathrm{~mol} \mathrm{~L}^{-1} \mathrm{de} \mathrm{Na}_{2} \mathrm{SO}_{4}$ contendo o agente dispersante "emulsogen" antes (Curva 1) e após a oxidação fotoeletrocatalítica, respectivamente. Ambos apresentam bandas largas em $540 \mathrm{~nm}$ (DPD) e $574 \mathrm{~nm}$ (DAD) (ver bandas inseridas nas Figuras1A e 1B) que podem ser atribuídas ao grupo azo presente como cromóforo nas moléculas dos corantes, que foram monitorados para se medir a remoção da cor durante a oxidação fotoeletrocatalítica. Uma banda intensa ao redor de $230 \mathrm{~nm}$, para ambos os corantes, é característica de máxima absorção do agente dispersante "emulsogen" presente como aditivo nas amostras dos corantes comercias e foi usada para monitoramento da remoção do dispersante.
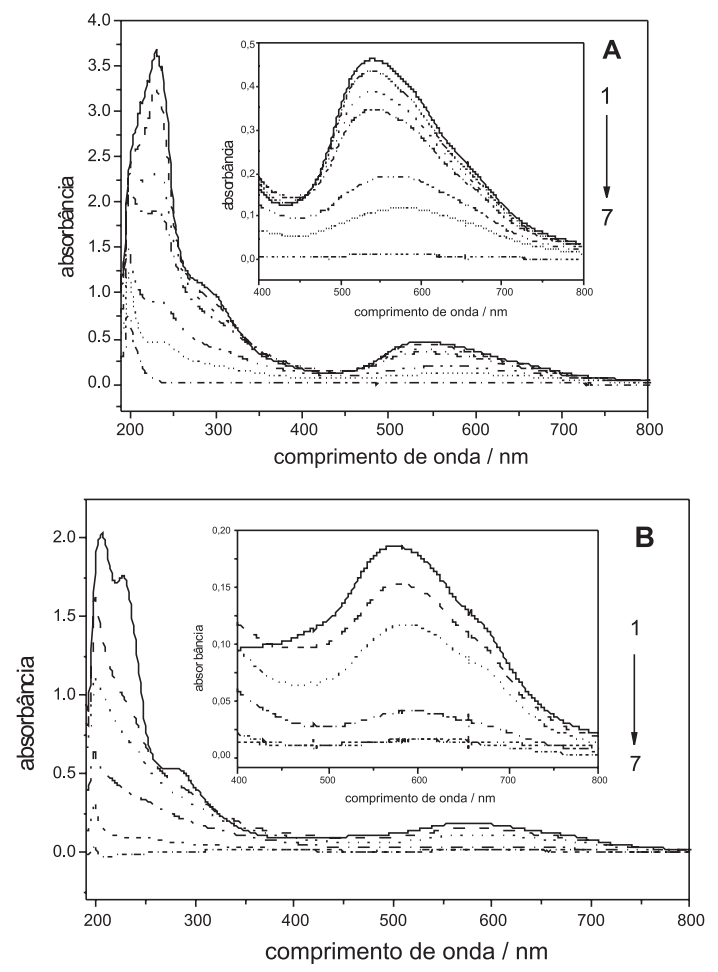

Figura 1. Espectros de absorção na região do UV-Visível para 50 ppm de corante preto dispersivo (A) e corante azul dispersivo (B) em $\mathrm{Na}_{2} \mathrm{SO}_{4} 0,1$ mol $L^{-1}$ submetido à oxidação fotoeletrocatalítica sobre eletrodo de $\mathrm{Ti} / \mathrm{TiO} \mathrm{O}_{2}$ irradiado com luz UV e sob potencial de 1,0 V durante: (1) 0, (2) 5, (3) 15 , (4) 30 , (5) 60, (6) 70, (7) $120 \mathrm{~min}$

Gráficos da variação da absorbância em função do tempo de oxidação fotoeletrocatalítica monitorando a descoloração de $250 \mathrm{~mL}$ de solução de cada corante sob potencial de $1,0 \mathrm{~V}$ e irradiação UV-Vis são mostrados nas Figuras 2A e 2B, respectivamente. Os resultados obtidos mostram $100 \%$ de remoção da cor de ambos os corantes preto e azul após 120 min (Curva 1). Adicionalmente, observa-se completa remoção do agente dispersante monitorado a $230 \mathrm{~nm}$ (Curva 2). Deste modo, estes resultados indicam que o processo de oxidação fotoeletrocatalítica pode ser um método efetivo para oxidar tanto o corante quanto o agente dispersante presente na solução.

Levando em consideração que os corantes dispersos podem ser mutagênicos em nível de traços, ${ }^{6,7}$ e se não forem completamente degradados podem permanecer na água e posteriormente serem acumulados nos sedimentos de solo, monitorou-se a seguir a eficiência da oxidação fotoeletrocatalítica pela análise cromatográfica com detector de arranjo 

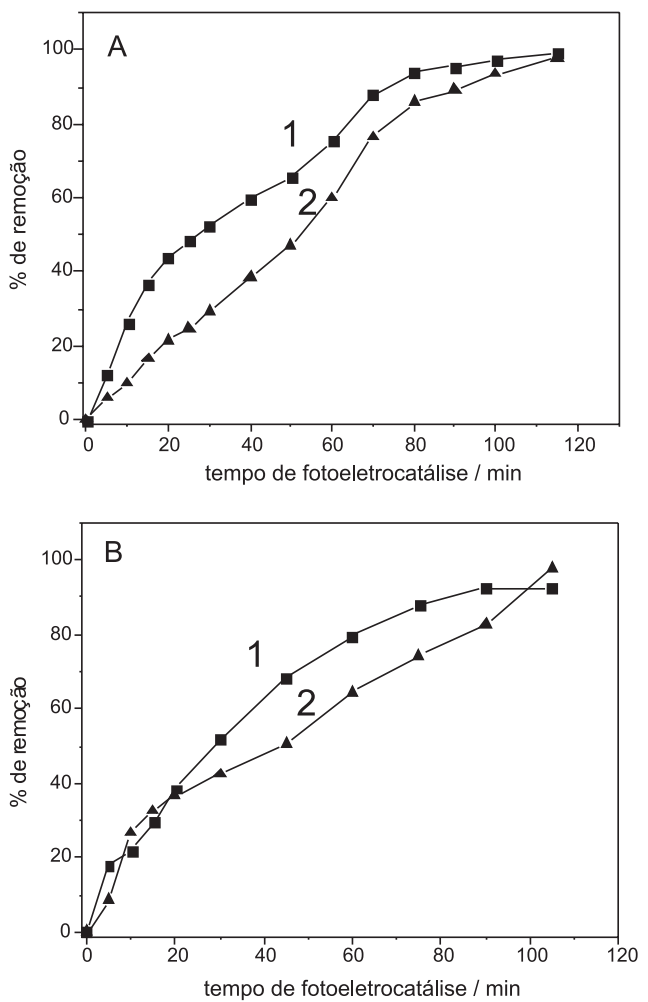

Figura 2. Percentagem de remoção da absorbância da solução de 50 ppm dos corantes: $(A)$ preto e $(B)$ azul dispersivo durante tratamento fotoeletrocatalítico e monitorados nos comprimentos de onda de $540 \mathrm{~nm}$ (1) e $574 \mathrm{~nm}$ (1) referente ao cromóforo e $230 \mathrm{~nm}$ (2) referente ao dispersante

de diodos previamente otimizada para cada uma das soluções de corante disperso contendo os corantes preto e azul dispersil.

\section{Análise cromatográfica dos corantes preto e azul dispersil}

Os parâmetros cromatográficos como tempo de retenção, fator de retenção constante $(\mathrm{k})$ e resolução do pico $(\mathrm{r})$ foram avaliados para cada espécie sob as condições experimentais propostas. Utilizaram-se as melhores condições obtidas em acetonitrila/água 80/20 (v/v), fluxo de $1,0 \mathrm{~mL} \mathrm{~min}^{-1}$ (resolução entre os picos $(\mathrm{r})>1,5$ ), tempo de análise em torno de 15 min e comprimento de onda de $570 \mathrm{~nm}$. A Figura 3

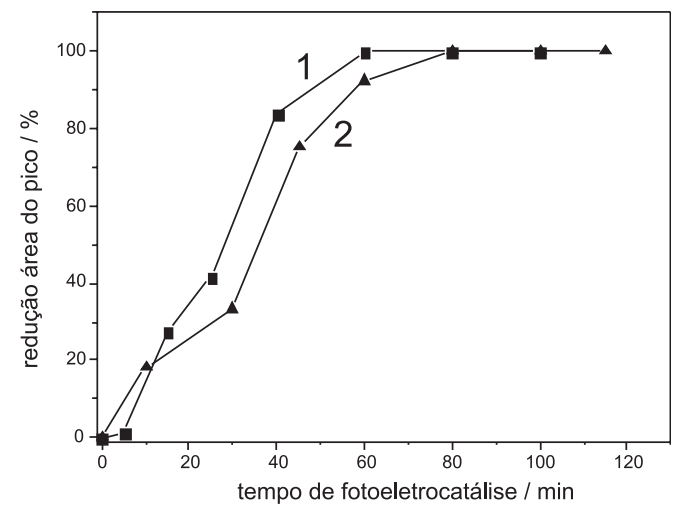

Figura 3. Percentagem de redução da área dos picos cromatográficos obtidos durante oxidação fotoeletrocatalítica sob tempo de retenção de (1) 5,3 min para o corante preto dispersivo e (2) 8,7 min para o azul dispersivo. Detector DAD, $\lambda=500 \mathrm{~nm}$. Fase móvel: acetonitrila: água 80:20. Fluxo: $1,0 \mathrm{~mL} \mathrm{~min}^{-1}$ mostra que a variação nas respectivas áreas de pico corresponde ao sinal cromatográfico registrado nos tempos de retenção de 5,3 min (Curva 1) e 8,7 min (Curva 2), respectivamente, para o corante preto e o azul dispersil. É possível observar que as áreas dos picos, para ambos os corantes, diminuem com a oxidação fotoeletrocatalítica, culminando no seu completo desaparecimento após $120 \mathrm{~min}$ de fotoeletrólise. Em adição, a análise do cromatograma base não exibe nenhum sinal cromatográfico para compostos intermediários com pico de retenção detectável no detector de arranjo de diodo operando em comprimentos de onda entre 200 a $800 \mathrm{~nm}$.

\section{Remoção de carbono orgânico total para os corantes preto e azul dispersil}

Considerando que nem sempre a total descoloração significa completa mineralização das substâncias aromáticas presentes na estrutura dos corantes e dispersantes, analisou-se a seguir o efeito da oxidação fotoeletrocatalítica das soluções de $50 \mathrm{ppm}$ de corante preto e azul dispersivo em $0,1 \mathrm{~mol} \mathrm{~L}^{-1}$ de $\mathrm{Na}_{2} \mathrm{SO}_{4}$ na remoção do teor de carbono orgânico total.

A Figura 4 apresenta os resultados da remoção de carbono orgânico total (COT) resultante da oxidação fotoeletrocatalítica de $50 \mathrm{ppm}$ de corante preto dispersil (DPD) (Curva 1) e $50 \mathrm{ppm}$ de corante azul dispersil (DAD) (Curva 2) em $0,1 \mathrm{~mol} \mathrm{~L}^{-1}$ de $\mathrm{Na}_{2} \mathrm{SO}_{4}$ durante $120 \mathrm{~min}$ sob potencial de $+1,0 \mathrm{~V}$ e irradiação UV. O máximo valor de COT removido foi 90 e $64 \%$ para os corantes DPD e DAD, respectivamente. Esses resultados indicam que embora a remoção da cor tenha alcançado $100 \%$, a remoção do COT é menor que a esperada para o DAD, mas os valores obtidos são muito maiores que o divulgado por outras técnicas. ${ }^{17-20}$

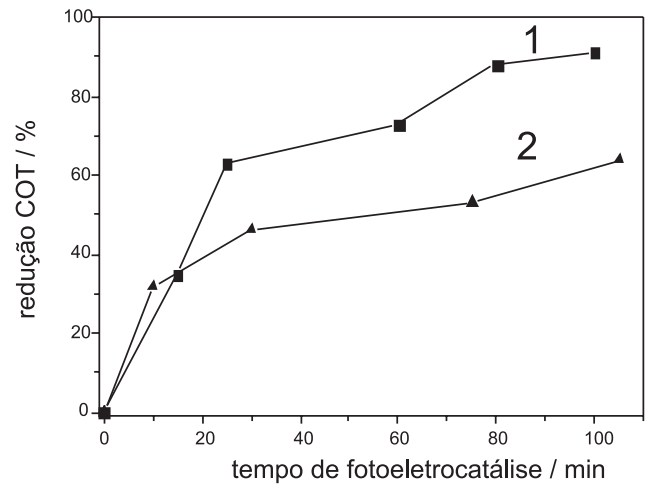

Figura 4. Redução de carbono orgânico total durante oxidação fotoeletrocatálitica de solução contendo 50 ppm de corantes dispersil preto (1) e corante dispersil azul (2) sob potencial de $+1 V$ e irradiação $U V$

Assim, a oxidação fotoeletrocatalítica poderia ser uma boa alternativa para promover a descoloração e a mineralização de efluentes da indústria têxtil contendo corantes dispersos. Dentro desse contexto o método foi aplicado para a degradação de um efluente gerado após etapa de tintura e lavagem da fibra, coletado em uma indústria têxtil de Farroupilha, RS.

\section{Tratamento de um efluente têxtil por oxidação fotoeletrocatalítica}

A Tabela 1 resume algumas das características físico-químicas da amostra de efluente coletado da indústria. Os respectivos espectros na região do UV-Visível obtidos antes e após a oxidação fotoeletrocatalítica do efluente são apresentados na Figura 5. O efluente não apresenta nenhuma banda na região do visível que possa ser identi- 
Tabela 1. Parâmetros físico-químicos correspondentes à oxidação fotoeletrocatálitica do efluente antes e após $300 \mathrm{~min}$ de tratamento sobre ânodos de $\mathrm{Ti} / \mathrm{TiO}_{2}$ e potencial de $+1,0 \mathrm{~V}$

\begin{tabular}{lcc}
\hline Parâmetros & Antes do tratamento & Após o tratamento \\
\hline \% clareamento & - & $60 \%$ \\
$\mathrm{DQO} / \mathrm{mg} \mathrm{L}^{-1}$ & 2347 & 1408 \\
$\%$ COT & - & $64 \%$ \\
$\mathrm{pH}$ & 6,1 & 7,6 \\
Condutividade/ $\mathrm{SS} \mathrm{cm}{ }^{-1}$ & 212 & 198 \\
Turbidez & 224 & 207 \\
\hline
\end{tabular}

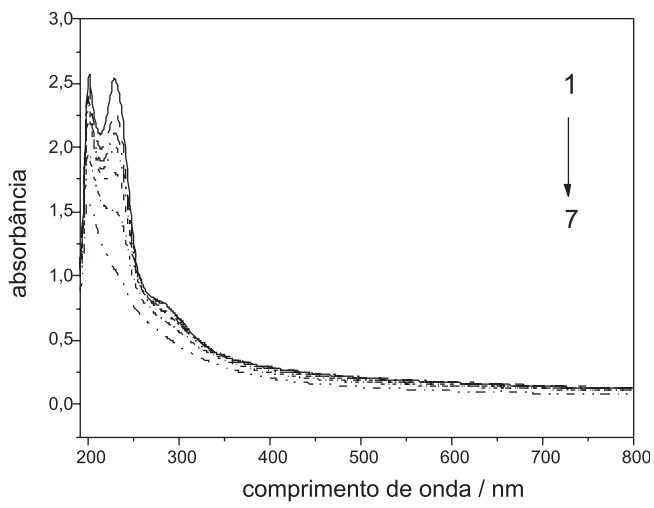

Figura 5. Espectros de absorção na região do UV-vis para o efluente da indústria têxtil corrigido com $\mathrm{Na}_{2} \mathrm{SO}_{4} 0,1 \mathrm{~mol} \mathrm{~L}^{-1}$ e submetido à oxidação fotoeletrocatalítica sobre eletrodo de $\mathrm{Ti} / \mathrm{TiO}$, irradiado com luz UV sob potencial de 1,0 V durante: (1) 0, (2) 5, (3) 10, (4) 15, (5) 40, (6) 90, (7) $300 \mathrm{~min}$

ficada como presença dos corantes preto e azul dispersil. Mas, exibe intensas bandas em $231 \mathrm{~nm}$ (dispersante), que diminui $60 \%$ após 300 min de fotoeletrólise. Observam-se ainda duas outras bandas em 203 e $288 \mathrm{~nm}$, que também diminuem na mesma intensidade em função do aumento do tempo da fotoeletrocatálise.

No entanto, as amostras do efluente submetidas à análise por cromatografia líquida de alta eficiência com detector de arranjo de diodos, usando as melhores condições experimentais definidas anteriormente, apresentam um pico bem definido em tempo de retenção de 5,2 min $(\mathrm{A}=2000 \mathrm{mAU}, \lambda=540 \mathrm{~nm})$ atribuído à ocorrência de resíduos do corante preto dispersil na amostra. Outro pico de grande intensidade foi observado em tempo de retenção de 2,2 min ( $\mathrm{A}=15000 \mathrm{mAU}, \lambda=$ $230 \mathrm{~nm}$ ), atribuído ao dispersante "emulsogen". A redução de ambos os picos durante a oxidação fotoeletrocatalítica pode ser acompanhada na Figura 6. Os resultados indicam uma redução de $100 \%$ do corante (Curva 1) e aproximadamente $60 \%$ do agente dispersante (Curva 2) após 300 min de oxidação fotoeletrocatalítica.

A degradação dos poluentes no efluente também foi confirmada pelas medidas de COT, como mostra a Figura 7. Após $30 \mathrm{~min}$ de fotoeletrocatálise já é possível ter uma redução do teor de carbono orgânico total bastante significativa (55\%), no entanto após 300 min de fotoeletrólise atingiu-se $64 \%$ de redução do teor de COT, indicando uma boa performance do método frente a outras técnicas descritas na literatura. ${ }^{18-22}$ Esses valores são confirmados pela redução de $60 \%$ no material orgânico monitorado pelas medidas da demanda química de oxigênio (DQO) que diminuem de 2347 para $1408 \mathrm{mg} \mathrm{L}^{-1}$ após 300 min de tratamento.

Do mesmo modo, é possível observar pela Tabela 1, que a oxidação fotoeletrocatalítica promove uma redução desprezível no valor da condutividade, pois também pode gerar alguns íons inorgânicos,

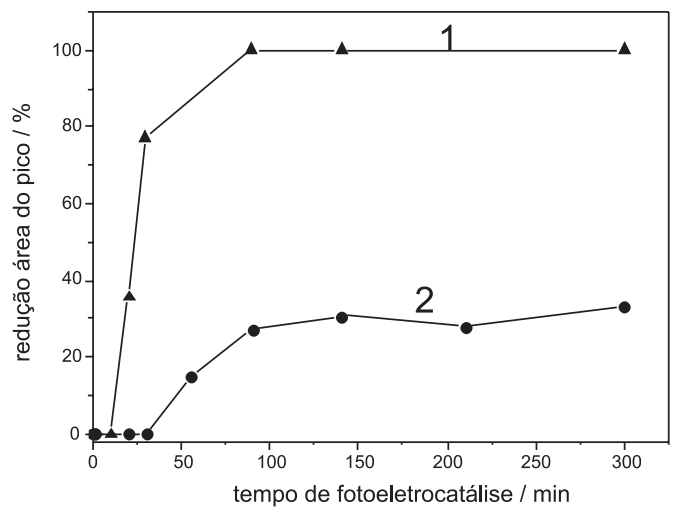

Figura 6. Percentagem de redução da área dos picos cromatográficos obtidos durante oxidação fotoeletrocatálitica do efluente da indústria têxtil para (1) 2,4 min para o dispersante $(\lambda=230 \mathrm{~nm})$ e (2) 5,3 min para o corante preto dispersil $(\lambda=500 \mathrm{~nm})$ Detector DAD. Fase móvel: acetonitrila: água 80:20. Fluxo: 1,0 $\mathrm{mL} \mathrm{min}^{-1}$

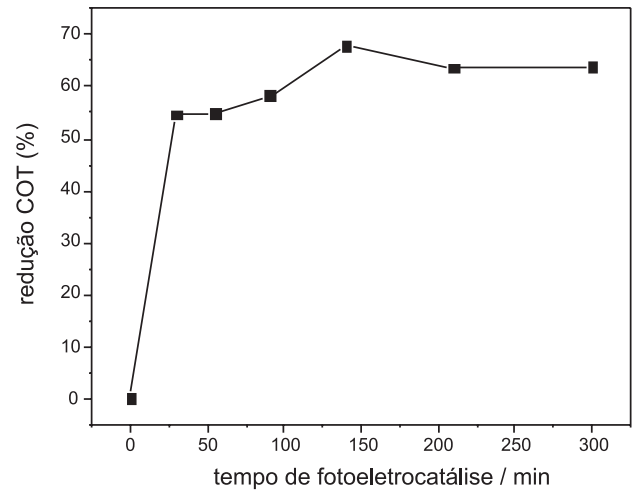

Figura 7. Redução do teor de carbono orgânico total durante tratamento fotoeletroquímico do efluente da indústria têxtil corrigido com $\mathrm{Na}_{2} \mathrm{SO}_{4} 0,1 \mathrm{~mol}$ $L^{-1}$ sobre eletrodo de $\mathrm{Ti} / \mathrm{TiO} \mathrm{O}_{2}$ irradiado com luz UV sob potencial de 1,0 V

como amônia e nitrato, que contribuem para a condutividade final da solução. Por outro lado, o valor do $\mathrm{pH}$ inicial da solução aumenta apenas ligeiramente de 6,1 para 7,6, o que pode ser explicado pela geração de íons hidroxilas geradas no cátodo após evolução de hidrogênio.

\section{CONCLUSÃO}

A oxidação fotoeletrocatalítica operando com irradiação UV e potencial de $+1,0 \mathrm{~V}$ no anodo nanoporoso de $\mathrm{Ti} / \mathrm{TiO}_{2}$ é um método de tratamento de efluente bastante eficiente para promover a degradação de corantes dispersos em solução aquosa contendo agentes dispersantes. O tratamento de $250 \mathrm{~mL}$ de solução contendo 50 ppm de cada um dos corantes preto dispersil e azul dispersil levou a $100 \%$ de descoloração da solução e 90 e 64\% de remoção de carbono orgânico total. O método foi aplicado com sucesso no tratamento do efluente coletado por uma indústria têxtil contendo baixa concentração desses corantes. Após 300 min de tratamento obteve-se $60 \%$ de redução da DQO e $64 \%$ de remoção de COT.

\section{AGRADECIMENTOS}

Ao CNPq, CAPES e FAPESP pelo suporte financeiro e a E. Witrew, Malhas Vêneto Ltda, Farroupilha, RS, Brasil pela valiosa colaboração no desenvolvimento do método e coleta das amostras. 


\section{REFERÊNCIAS}

1. Kunz, A.; Peralta-Zamora, P.; Moraes, S. G.; Durán, N.; Quim. Nova 2002, 25, 78.

2. Zollinger, H.; Color chemistry: syntheses, properties and applications of organic dyes and pigments, $2^{\text {nd }}$ ed., VCH Publishers: New York, 1991.

3. Gonçalves, M. S. T.; Pinto, E. M. S.; Nkeonye, P.; Oliveira-Campos, A. M. F.; Dyes Pigm. 2005, 64, 135.

4. Golob, V.; Tusek, L.; Dyes Pigm. 1999, 40, 211.

5. CPRH - Companhia Pernambucana do Meio Ambiente; Roteiro Complementar de licenciamento e fiscalização para a tipologia têxtil, CPRH: Recife, 2001.

6. Oliveira, D. P.; Carneiro, P. A.; Rech, C. M.; Zanoni, M. V. B.; Umbuzeiro, G. A.; Environ. Sci. Technol. 2006, 40, 6682.

7. Oliveira, D. P.; Carneiro, P. A.; Sakagami, M. K.; Zanoni, M. V. B.; Umbuzeiro, G. A.; Mutat. Res. Genetic Toxicol. Environ. Mutagen. 2007, $626,135$.

8. Kim, D. H.; Anderson, M. A.; Environ. Sci. Technol. 1994, 28, 479.

9. Candal, R. J.; Zeltner, W. A.; Anderson, M. A.; Environ. Sci. Technol. 2000, 34, 3443.

10. Candal, R. J.; Zeltner, W. A.; Anderson, M. A.; J. Adv. Oxidation Technol. 1998, 3, 270 .
11. Candal, R. J.; Zeltner, W. A.; Anderson, M. A.; J. Environ. Eng. 1999, 125, 906.

12. Minero, C.; Mariella, G.; Maurino, V.; Vione, D.; Pelizzetti, E.; Langmuir 2000, 16, 8964

13. Zanoni, M. V. B.; Sene, J. J.; Anderson, M. A.; J. Photochem. Photobiol. 2003, 157, 55

14. Carneiro, P. A.; Osugi, M. E.; Sene, J. J.; Anderson, M. A.; Zanoni, M. V. B.; Electrochim. Acta 2004, 49, 3807.

15. Selcuk, H.; Sene, J. J.; Zanoni, M. V. B.; Sarikaya, H. Z.; Anderson, M. A.; Chemosphere 2004, 54, 969.

16. Osugi, M. E.; Umbuzeiro, G. A.; Anderson, M. A.; Zanoni, M. V. B.; Electrochim. Acta 2005, 50, 5261.

17. Chu, W.; Ma, C. W.; Water Res. 2000, 34, 3153.

18. Bertazzoli, R.; Pelegrini, R.; Quim. Nova 2002, 25, 477.

19. Green, J. M.; Sakol, C.; Am. Dyest. Rep. 1985, 74, 50.

20. Arslan, I.; J. Hazard. Mater. B 2001, 85, 229.

21. Alaton, I. A.; Dyes Pigm. 2004, 60, 167.

22. Szpyrkowicz, L.; Juzzolino, C.; Kaul, S. N.; Water Res. 2001, 35, 2129.

23. Clesceri, L. S.; Greenberg, A. E.; Eaton, A. D.; Standard Methods for the Examination of Water and Wastewater, $17^{\text {th }}$ ed., APNA: Washington, 1989. 